\title{
Using the BODE Index and Comorbidities to Predict Health Utilization Resources in Chronic Obstructive Pulmonary Disease
}

This article was published in the following Dove Press journal: International Journal of Chronic Obstructive Pulmonary Disease

\author{
Chin-Ling $\operatorname{Li} \mathbb{D}^{1,2}$ \\ Mei-Hsin Lin' \\ Pei-Shiuan Chen' \\ Yuh-Chyn Tsai ${ }^{1,2}$ \\ Lien-Shi Shen ${ }^{1,2}$ \\ Ho-Chang Kuo (D) ${ }^{1,3,4}$ \\ Shih-Feng Liu ${ }^{1,4,5}$
}

'Department of Respiratory Therapy, Kaohsiung Chang Gung Memorial Hospital, Kaohsiung City, Taiwan; ${ }^{2}$ Department of Healthcare

Administration and Medical Informatics, Kaohsiung Medical University, Kaohsiung City, Taiwan; ${ }^{3}$ Department of Pediatrics, Kaohsiung Chang Gung Memorial Hospital, Kaohsiung City, Taiwan; ${ }^{4}$ Chang Gung University College of Medicine, Taoyuan, Taiwan; ${ }^{5}$ Department of Internal Medicine, Division of Pulmonary and Critical Care Medicine, Kaohsiung Chang Gung Memorial Hospital, Kaohsiung City, Taiwan
Correspondence: Shih-Feng Liu Division of Pulmonary \& Critical Care Medicine, Department of Internal Medicine, Department of Respiratory Therapy, Kaohsiung Chang Gung Memorial Hospital and Chang Gung University College of Medicine, Taiwan, No. 123, Ta-Pei Road, Niaosong District, Kaohsiung 833, Taiwan

Tel +88677317123 ext. 8199

Fax +8867732 24942

Email liuphysico@yahoo.com.tw
Background and Objective: Chronic Obstructive Pulmonary Disease (COPD) is a common chronic respiratory disease that in the long term may develop into respiratory failure or even cause death and may coexist with other diseases. Over time, it may incur huge medical expenses, resulting in a heavy socio-economy burden. The BODE (Body mass index, airflow Obstruction, Dyspnea, and Exercise capacity) index is a predictor of the number and severity of acute exacerbations of COPD. This study focused on the correlation between the BODE index, comorbidity, and healthcare resource utilization in COPD.

Patients and Methods: This is a retrospective study of clinical outcomes of COPD patients with complete BODE index data in our hospital from January 2015 to December 2016. Based on the patients' medical records in our hospital's electronic database from January 1, 2015 to August 31, 2017, we analyzed the correlation between BODE index, Charlson comorbidity index (CCI), and medical resources.

Results: Of the 396 patients with COPD who met the inclusion criteria, 382 (96.5\%) were male, with an average age of $71.3 \pm 8.4$ years. Healthcare resource utilization was positively correlated with the BODE index during the 32 months of retrospective clinical outcomes. The study found a significant association between the BODE index and the CCI of COPD patients $(p<0.001)$. In-hospitalization expenses were positively correlated with CCI ( $p<$ 0.001). Under the same CCI, the higher the quartile, the higher the hospitalization expenses. BODE quartiles were positively correlated with number of hospitalizations $(p<0.001)$, hospitalization days $(p<0.001)$, hospitalization expenses $(p=0.005)$, and total medical expenses $(p=0.024)$.

Conclusion: This study demonstrates the value of examining the BODE index and comorbidities that can predict healthcare resource utilization in COPD.

Keywords: chronic obstructive pulmonary disease, BODE index, Charlson comorbidity index, medical burden, 6 min walk test

\section{Introduction}

Chronic Obstructive Pulmonary Disease (COPD) is a common chronic respiratory disease characterized by progressive and irreversible airflow limitation. Symptoms of COPD may include dyspnea, chronic cough, productive cough, weight loss, and decreased exercise and daily activity tolerance. Hypoxia occurs when blood oxygen levels drop during rest, sleep, or activity, and it may develop into respiratory failure after recurrent exacerbations. COPD often coexists with other diseases; comorbidities are common among COPD patients at any stage of the disease. ${ }^{1,2}$ 
The Charlson comorbidity index (CCI) was first developed by Charlson et al in 1987 as an assessment of disease severity and mortality prediction. It is a criterion of 15 chronic diseases, including myocardial infarction, chronic heart failure, COPD, peripheral vascular disease, cerebrovascular disease, dementia, diabetes mellitus, systemic hypertension, liver disease, renal disease, cancer, connective tissue diseases, HIV, skin ulcers, peptic ulcer disease, depression and anticoagulants use. ${ }^{3}$ CCI scores comorbidity on a scale of $0-33$, where higher CCI indicates a greater number and severity of comorbidity with higher medical needs that lead to a higher medical burden. ${ }^{4-7}$ Multiple comorbidities are common among COPD patients; as a consequence, there is a correlation between the CCI and the mortality rate ${ }^{8}$ and hospitalization days ${ }^{9}$ of COPD patients.

At present, many tools are used to assess the severity and prognosis of COPD patients. Celli et al introduced the BODE index, which has clinically proven to be a significant predictor of disease severity and mortality. ${ }^{10}$ The BODE index is a multidimensional index of disease severity in COPD that incorporates four independent predictors: the body mass index (BMI), the degree of airflow obstruction assessed by the Forced Expiratory Volume in one second $\left(\mathrm{FEV}_{1}\right)$, the modified Medical Research Council (mMRC) dyspnea scale, and the exercise capacity assessed by the 6-min walking distance (6MWD) test. Among patients with COPD, the BODE index is a better predictor of disease severity, number of acute exacerbations, and mortality than the $\mathrm{FEV}_{1}{ }^{11}$ Saint George's Respiratory Questionnaire (SGRQ) is also an instrument evaluating COPD patients that provides information on health status (quality of life). Studies have shown that the BODE index better predicts mortality than the SGRQ. ${ }^{5}$

The score of the BODE index is divided into four quartiles, ${ }^{10}$ where the highest quartile indicates the most serious disease. Increasing BODE quartiles were associated with a higher risk of death. ${ }^{12,13}$ Except for survival predictions, the BODE quartile is a good predictor of both the number and severity of exacerbations in patients with COPD. ${ }^{11}$ Acute exacerbation of COPD is the main cause of increasing hospitalization ${ }^{13}$ and medical needs, accounting for most of the economic burden of the disease. The purpose of this study was to analyze the correlation between the BODE index, comorbidity, and healthcare resource utilization in COPD.

\section{Methods and Materials Data Source}

This is a retrospective study of clinical outcomes. The data were drawn from the electronic database of the Kaohsiung Chang Gung Medical Center (KCGH) and were reviewed and approved by the Institutional Review Board Committee (IRB: 201701293B0) of Chang Gung Medical Center. The IRB of Chang Gung Medical Center did not require patients to agree to a review of their medical records for this retrospective study. This study complies with the Helsinki Declaration and Good Clinical Practice Guidelines and was approved by the Ethics Committee of Chang Gung Medical Center.

Patients who had undergone a 6 min walking test (6MWT) in the respiratory therapy department $(\mathrm{n}=$ 1063) between 2015 and 2016 were selected for data collection and analysis. The patients' retrospective reports of the 6 min walking test were dated from January 31, 2015, to August 31, 2017 (a total of 32 months). The electronic data of the medical records and healthcare resource utilization were from KCGH. The inclusion criteria of patients with COPD in KCGH were as follows: 1) diagnostic code ICD-9-CM: 490-496, ICD-10-CM: J41$\mathrm{J} 44(\mathrm{n}=507) ; 2)$ at least a complete clinical record of 6MWT; 3) diagnosed as COPD by a post-bronchodilator FEV1/FVC $<70 \%$ with persistent expiratory flow obstruction according to the COPD GOLD guideline; 4) excluded those who were less than 40 years old. We collected medical information, the number of outpatient visits, the number and days of hospitalization, and medical expenses of the selected patients $(\mathrm{n}=396)$. The selection process was shown below (Figure 1).

\section{Study Population}

Following Celli et al, the BODE index was categorized into 4 quartiles: quartile 1 by a score of $0-2$; quartile 2 , 3-4; quartile 3, 5-6; and quartile 4, 7-10. ${ }^{10,13}$ The CCI data were obtained from the specific diagnostic codes that appeared during the 32 months of the patients' retrospective reports. The data on healthcare resource utilization consisted of total medical expenses including outpatient clinic visits, hospitalization days, examination fees, and drug costs during the 32 months of each patient's retrospective report. The correlations between the BODE index, CCI, and medical expenses in patients with COPD were then analyzed. 


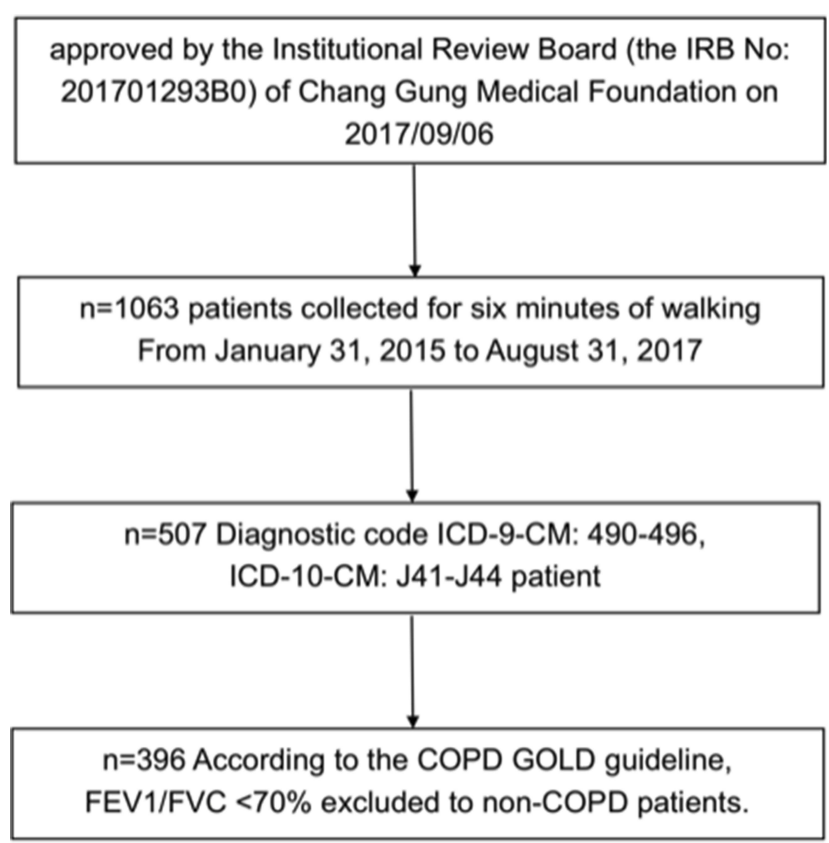

Figure I Flow chart of participant selection in this study.

\section{Statistical Analysis}

Data distributions are presented as mean and standard deviation (mean $\pm \mathrm{SD}$ ), median (Interquartile range, IQR), or N (\%). Chi-square tests, Fisher's exact test, and one-way analysis of variance (one-way ANOVA) were applied to compare the differences in variables by quartiles, and then a posteriori comparisons were made with Scheffé's test. Lastly, one-way ANOVA and linear contrasts were applied to check for linear trends. Analysis of data was performed using the Statistical Package for the Social Sciences (SPSS) version 22.

\section{Results}

A total of 396 COPD patients were included under the inclusion criteria. Table 1 shows the baseline characteristics of the study population. A majority of the subjects, 382 were male, accounting for $96.5 \%$ of the total, and the average age was 71.3 years \pm 8.4 years. In terms of smoking status, the average length of smoking was 31.7 years \pm 18.5 years. The body mass index (BMI, $\mathrm{Kg} / \mathrm{m}^{2}$ ) was $23.5 \pm 4.1$.

According to the definition of severity of the COPD GOLD guideline, ${ }^{14,15}$ the patients with moderate to severe severity made up $82.6 \%$, the largest subset in this study. Among them, there were 187 patients with moderate severity, making up $47.2 \%$ of the total, and 140 patients with severe severity, making up $35.4 \%$ of the total. CCI
Table I Baseline Characteristics of COPD Patients Included in the Study $(n=396)$

\begin{tabular}{|l|l|}
\hline Factors & Mean \pm SD or N (\%) \\
\hline Age (yr) & $73.1 \pm 9.5$ \\
Male (\%) & $382(96.5)$ \\
Smoking history (pack-yr) & $31.7 \pm 18.5$ \\
Body mass index (BMI) & $23.5 \pm 4.1$ \\
FVC (\% of predicted value) & $79.7 \pm 16.7$ \\
FEVI/FVC (\%) & $52.7 \pm 10.6$ \\
FEVI (\% of predicted value) & $55.2 \pm 18.2$ \\
DLCO (\%) & $68.5 \pm 21.0$ \\
\hline GOLD Stage (\%) & \\
GOLD I & $46(11.6)$ \\
GOLD II & $187(47.2)$ \\
GOLD III & $140(35.4)$ \\
GOLD IV & $23(5.8)$ \\
\hline MIP & $72.2 \pm 30.5$ \\
MEP & $98.3 \pm 46.8$ \\
CCI & $3.3 \pm 2.8$ \\
BODE index & $3.0 \pm 2.1$ \\
\hline BODE Quartile: QI, Q2, Q3, Q4 (\%) & \\
Quartile I & $188(47.5)$ \\
Quartile 2 & $109(27.5)$ \\
Quartile 3 & $71(17.9)$ \\
Quartile 4 & $28(7.1)$ \\
\hline mMRC & $1.72 \pm 0.9$ \\
\hline mMRC Dyspnea Scale & $25 / 133 / 173 / 56 / 9$ \\
Scale 0/I/2/3/4 & $351.9 \pm 111.6$ \\
\hline 6MWD (m) & \\
\hline
\end{tabular}

Notes: auartile I was defined by a score of $0-2$, quartile 2 by a score of 3-4, quartile 3 by a score of $5-6$, and quartile 4 by a score of 7-10.

Abbreviations: $\mathrm{BMI}$, body mass index; $\mathrm{CCl}$, Charlson comorbidity index; 6MWD, 6 mins walking distance; FEVI, forced expiratory volume in I second; FVC, forced vital capacity; MRC score, Medical Research Council dyspnea scale; BODE index, composite index of Body mass index, airflow maximum expiratory pressure Obstruction, Dyspnea, and Exercise capacity; MIP, maximum inspiratory pressure; MEP, maximum expiratory pressure.

had a mean score of $3.3 \pm 2.8 ;$ mMRC, $1.72 \pm 0.9$; and the BODE index, $3.3 \pm 2.1$ (quartile 1: $47.5 \%(n=188)$; quartile 2: $27.5 \%(\mathrm{n}=109)$; quartile $3: 17.9 \%(\mathrm{n}=71)$; quartile $4: 7.1 \%(\mathrm{n}=28))$. The mean distance of $6 \mathrm{MWT}$ was $351.9 \pm 11.6 \mathrm{~m}$ (Table 1).

Figure 2 shows the correlation between the BODE index and the CCI index. The higher the BODE quartile score, the higher the CCI score, and the positive correlation between the two was significant $(p<0.001)$. In addition, $\mathrm{CCI}$ was divided into two groups: high $(\mathrm{CCI} \geq 3)$ and low $(\mathrm{CCI}<3)$. Because BODE quartile $3(\mathrm{n}=71)$ and quartile $4(\mathrm{n}=28)$ had fewer samples, we combined them 


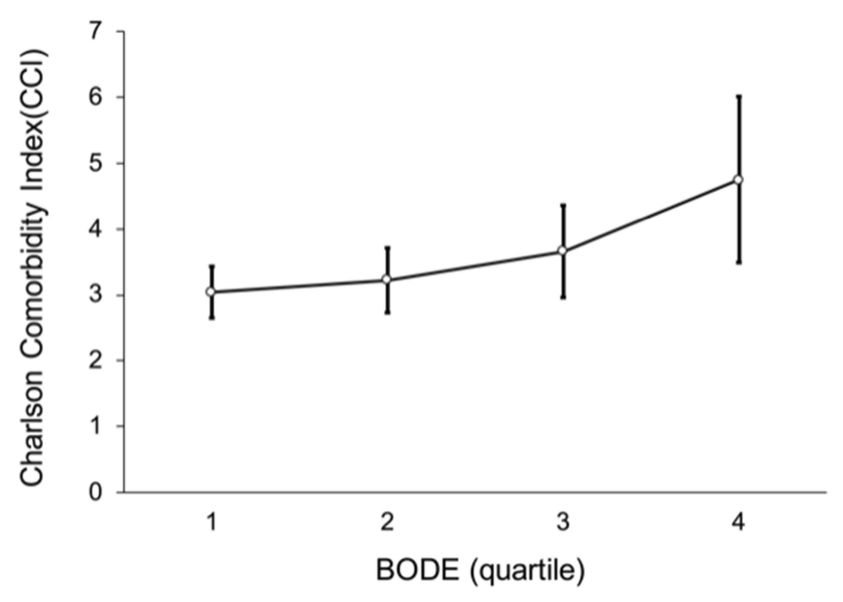

Figure 2 Line of positive correlation between BODE quartiles and Charlson comorbidity index $(p<0.001)$.

Notes: Post hoc comparison with Scheffé's method for significant difference between BODE quartile I and quartile $4(p=0.024)$.

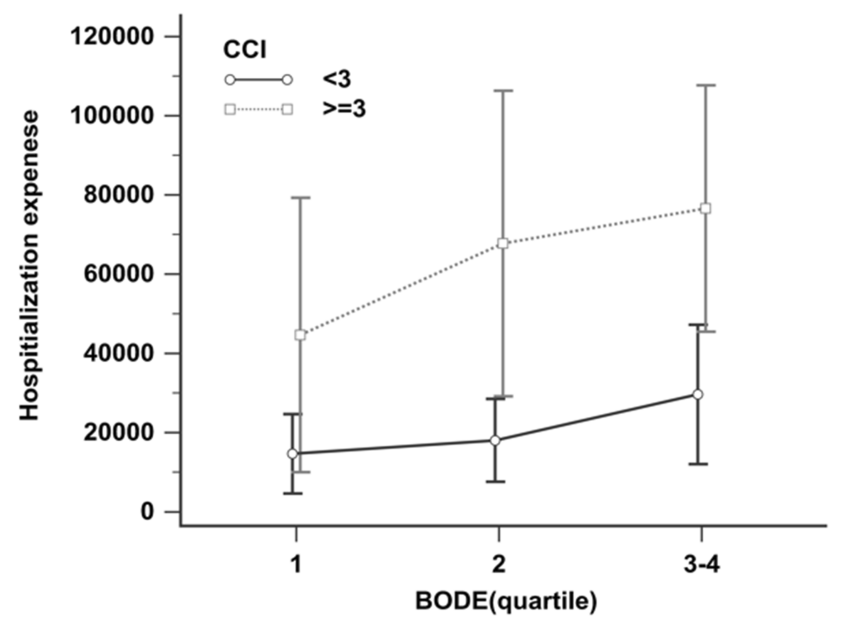

Figure 3 Hospitalization expenses by BODE quartiles for the high $\mathrm{CCl}$ and low $\mathrm{CCl}$ groups. Patients with high $\mathrm{CCl}$ level had higher hospitalization expenses than those with low $\mathrm{CCl}$ level after adjusting for BODE quartiles $(p<0.00 \mathrm{l})$.

to avoid statistical error (Figure 3). Figure 3 shows BODE against hospitalization expenses for the high CCI and low CCI groups. Hospitalization expenses were positively correlated with CCI $(p<0.001)$. At the same CCI, the higher the quartile, the higher the hospitalization expenses. In the high CCI group, quartile 1 hospitalization expenses were NT\$ 44,664; quartile 2 hospitalization expenses, NT\$ 67,745; and quartiles 3-4 hospitalization expenses, NT\$ 76,589 ; thus, the average hospitalization expenses increased, but not statistically significantly so. In the low CCI group, the hospitalization expenses were quartile 1, NT\$14,643; quartile 2, NT\$ 18,030; and quartiles 3-4, NT $\$ 29,638$. Again, the average hospitalization expenses increased, but not statistically significantly so.

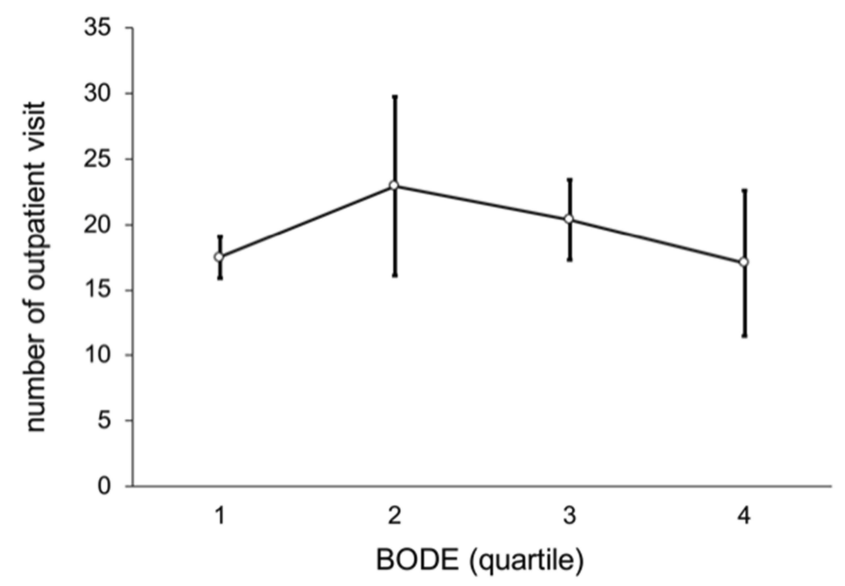

Figure 4 Non-linear trend of number of outpatient visits by BODE quartiles ( $p=$ $0.462)$.

In these patients as a whole, the average number of outpatient visits per person was 16 (median; IQR, 10-23), and outpatient medical expenses were NT\$45,393. The number of hospitalizations was $0(0-1)$, and hospitalization days were $0(0-12)$. Figure 4 shows a non-linear trend among BODE quartiles by number of outpatient visits $(p=0.462)$, such that there were more outpatient visits in BODE quartile 2 than quartiles $3-4$. Figures 5 and 6 show a linear trend of BODE quartiles by number of hospitalizations and of BODE quartiles by length of hospital stay, with both trends significant $(p<0.001)$. Both of these relationships (BODE quartiles with number of hospitalizations and length of hospital stay) are significant after post hoc comparison using Scheffé's method such that BODE quartile $1=$ quartile $2<$ quartile 3 $<$ quartile 4 . Thus, the higher BODE quartiles showed significantly greater hospitalization needs. The number of

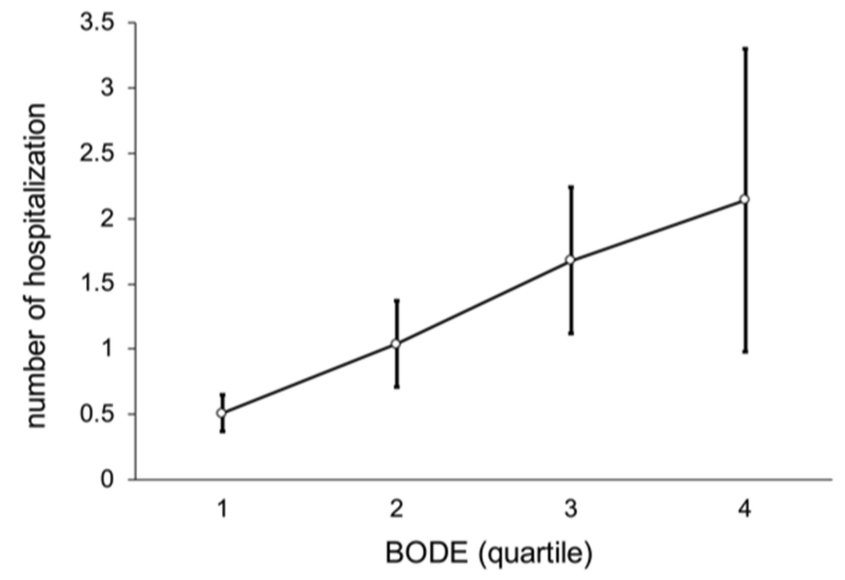

Figure 5 Linear trend of number of hospitalizations by BODE quartile $(p<0.00 \mathrm{I})$. Notes: The relation among BODE quartiles by number of hospitalizations after post hoc comparison with Scheffé's method is BODE quartile 1 = quartile $2<$ quartile $3<$ quartile 4. 


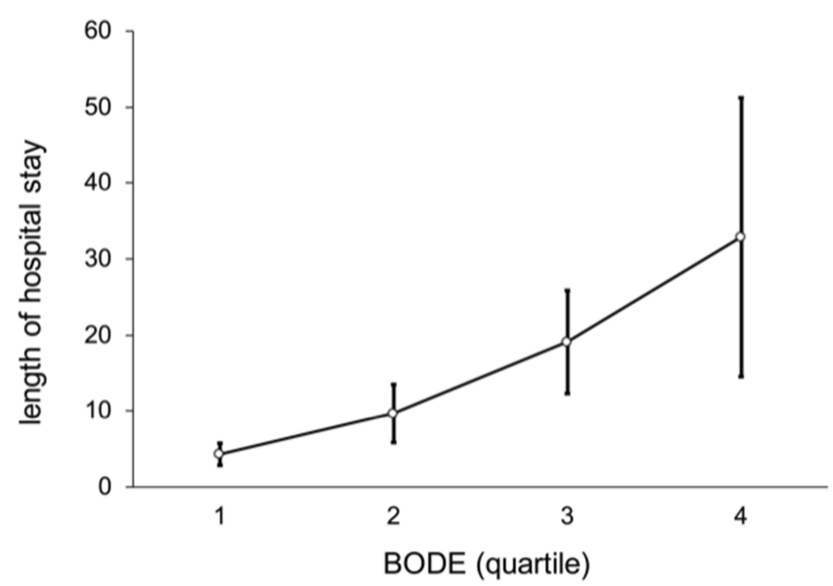

Figure 6 Linear trend of length of hospital stay by BODE quartile $(p<0.001)$. Notes: The relation among BODE quartiles for length of hospital stay after post hoc comparison with Scheffeé's method is BODE quartile I = quartile 2 < quartile 3 $<$ quartile 4 .

hospitalizations for BODE quartile 3 were 1.17 times more hospitalizations than quartile $1(p<0.001)$. Quartile 4 patients were admitted 13.79 days longer on average than quartile 3 ( $p=0.042)$, a significant difference.

Figure 7 shows a non-linear trend among BODE quartiles for outpatient expenses $(p=0.532)$, such that the outpatient expenses of BODE quartile 2 were NT\$ 78,475 , higher than BODE quartile 3, NT\$ 66,473 and quartile 4 NT\$ 54,023. Figure 8 shows the relation between BODE quartiles and hospitalization; the overall $p$-value was marginally significant $(p=0.047)$ after multiple comparisons. Figure 9 shows a linear trend among BODE quartiles for total medical expenses $(p=0.024)$.

\section{Discussion}

In this study, the data show the BODE index to be correlated positively with the CCI, number of hospitalizations,

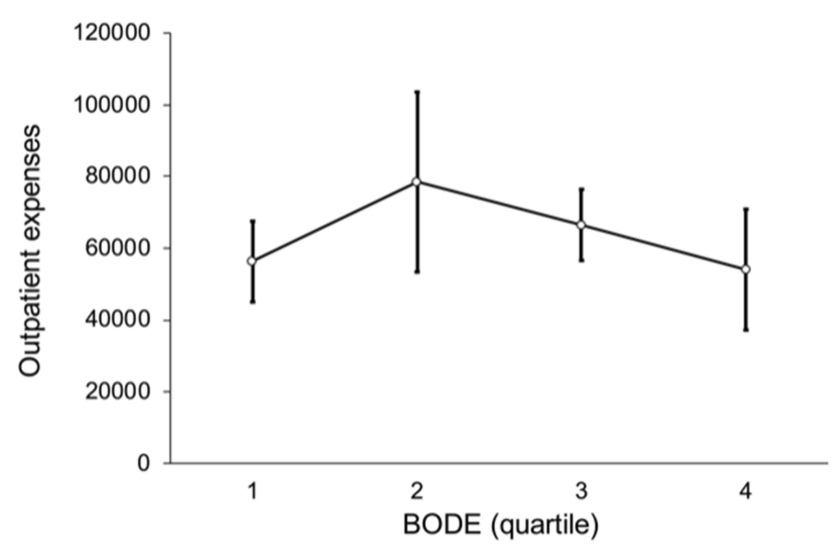

Figure 7 Non-linear trend of outpatient expenses by BODE quartile $(p=0.532)$.

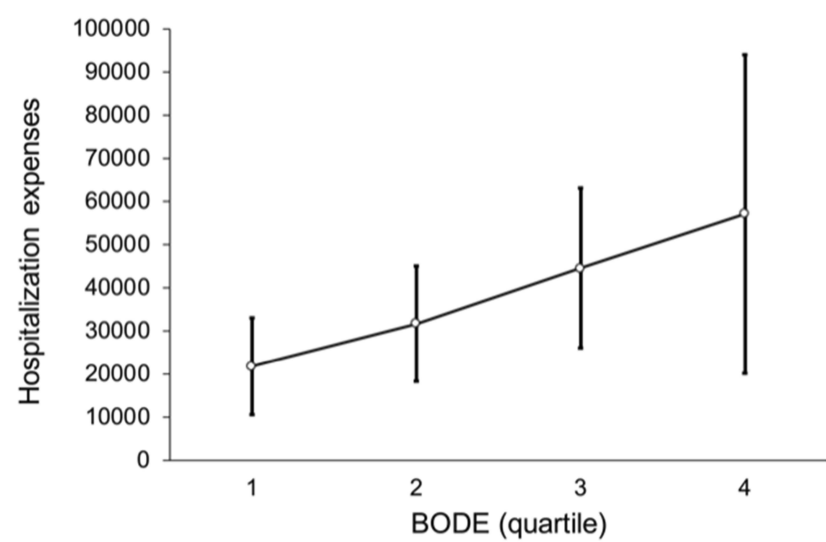

Figure 8 Relation between BODE quartiles and hospitalization. Overall $p$-value is marginally significant $(p=0.047)$ after multiple comparisons.

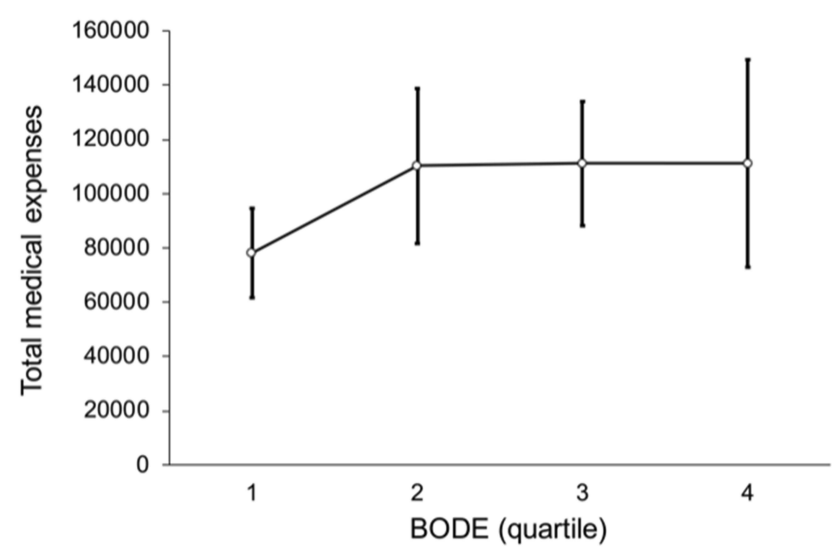

Figure 9 This shows linear trend among BODE quartiles with total medical expenses $(p=0.024)$

length of hospital stay, and total medical expenses of COPD patients. For the same CCI, the higher the BODE quartile, the higher the hospitalization expenses.

Why is CCI correlated with medical expenses? This is because of the many comorbidities in COPD, which as a chronic inflammatory disease of the lungs causes inflammatory mediators to spill over into the blood stream and injure other organs. Other studies have found that COPD patients had more comorbidities. ${ }^{16,17}$ "High CCI will have higher medical costs," Cavaillès et al found, stating that "COPD comorbidities lead to more hospitalization, mortality and medical costs" and "Hospitalization cost is the major cost." ${ }^{18-20}$ This study also found CCI scores to be associated with hospitalization expenses.

$\mathrm{FVC} / \mathrm{FEV}_{1}$ is a tool used to diagnose the severity of $\mathrm{COPD}^{21}$ airflow, but does not suffice to measure the overall severity of COPD. The BODE index is an effective multidimensional-grading-system tool that is better predictive of 
disease severity, hospitalization needs, and risk of death than $\mathrm{FEV}_{1 .}{ }^{13,22,23}$

Previous research found that a worse BODE index is associated with higher healthcare resource utilization. ${ }^{24}$ The integrated Pro-BODE score proved proportional to the cost of care. ${ }^{25}$ In their study, Bu et al found that "Patients with increased BODE index had significantly higher hospital presentation rates and longer total beddays compared to those with stable BODE index." ${ }^{26}$ Both the frequency of hospitalization and the number of hospital stays increased with a higher BODE index, which is consistent with our research.

In addition, according to our data, patients with quartile 3 and quartile 4 BODE scores have fewer outpatient medical needs than quartile 2 . We speculate that the likely reasons are as follows: 1) Patients with BODE quartile 3 and quartile 4 have high disease severity that may cause a higher frequency of hospitalization and longer hospitalization stays, which leads to a decreased number of outpatient visits. 2) The above-average symptoms of dyspnea and reduced mobility of patients with BODE quartile 3 and quartile 4 may increase the difficulty of visiting outpatient clinics, as they need more assistive devices such as wheelchairs, oxygen, and caregiver assistance to go out to fulfill their medical needs, leading to a lower outpatient clinical visit rate than in patients with quartile 2 BODE scores.

\section{Limitations}

There were some limitations to our study. First, as this is a retrospective study, a prospective study is needed to prove the clinical outcomes. Second, the source of the case data was a single medical center, and the cases who performed the 6MWT were almost all male during the period covered by our data collection, which cannot allow a more objective, representative selection of all groups of COPD. Third, medical expenses are divided into direct medical expenses and indirect medical expenses. The data we collected are only of direct medical expenses. Our study had a lack of indirect medical expense data related to COPD and comorbidities. These indirect medical expenses include the cost of productivity loss at work, the loss of productivity due to early retirement, payments for disability pensions, and care fees. Theoretically, patients with higher BODE will have higher indirect medical expenses, leading the total medical costs in higher BODE index patients to be higher than in those with lower BODE.

\section{Conclusion}

The study demonstrates that the BODE index is positively correlated with the number of hospitalizations, hospitalization days, hospitalization expenses, and total medical expenses. With the same CCI, the higher the Quartile, the higher the hospitalization expenses. Therefore, the BODE index and CCI together can accurately predict the medical expenses of COPD.

\section{Acknowledgments}

We would like to express our sincere gratitude to the Biostatistics Center, Kaohsiung Chang Gung Memorial Hospital, for statistical work.

\section{Disclosure}

The authors report no conflicts of interest in this work.

\section{References}

1. Negewo NA, Gibson PG, McDonald VM. COPD and its comorbidities: impact, measurement and mechanisms. Respirology. 2015;20: 1160-1171. doi:10.1111/resp. 12642

2. Divo M, Cote C, de Torres JP, et al. Comorbidities and risk of mortality in patients with chronic obstructive pulmonary disease. Am J Respir Crit Care Med. 2012;186:155-161. doi:10.1164/rccm. 201201-0034OC

3. Charlson ME, Pompei P, Ales KL, MacKenzie CR. A new method of classifying prognostic comorbidity in longitudinal studies: development and validation. J Chronic Dis. 1987;40:373-383. doi:10.1016/ 0021-9681(87)90171-8

4. Charlson ME, Charlson RE, Peterson JC, Marinopoulos SS, Briggs WM, Hollenberg JP. The Charlson comorbidity index is adapted to predict costs of chronic disease in primary care patients. $J$ Clin Epidemiol. 2008;61:1234-1240. doi:10.1016/j.jclinepi.2008.01.006

5. Marin JM, Cote CG, Diaz O, et al. Prognostic assessment in COPD: health related quality of life and the BODE index. Respir Med. 2011;105:916-921. doi:10.1016/j.rmed.2011.01.007

6. Mannino DM, Higuchi K, Yu T-C, et al. Economic burden of COPD in the presence of comorbidities. Chest. 2015;148:138-150. doi:10.13 78/chest.14-2434

7. Simon-Tuval T, Scharf SM, Maimon N, Bernhard-Scharf BJ, Reuveni H, Tarasiuk A. Determinants of elevated healthcare utilization in patients with COPD. Respir Res. 2011;12:7. doi:10.1186/1465-99 21-12-7

8. Almagro P, Calbo E, de Echaguïen AO, et al. Mortality after hospitalization for COPD. Chest. 2002;121:1441-1448. doi:10.1378/chest. 121.5.1441

9. Buhr RG, Chen X, Tashkin DP, Ong MK. Readmissions For COPD and the Interaction of Hospital Type and Case Mix Comorbidity. D13 the Revolving Door: Copd Hospitalization and Readmission. American Thoracic Society; 2017:A7001-A.

10. Celli BR, Cote CG, Marin JM, et al. The body-mass index, airflow obstruction, dyspnea, and exercise capacity index in chronic obstructive pulmonary disease. $N$ Engl J Med. 2004;350:1005-1012. doi:10. 1056/NEJMoa021322

11. Marin JM, Carrizo SJ, Casanova C, et al. Prediction of risk of COPD exacerbations by the BODE index. Respir Med. 2009;103:373-378. doi:10.1016/j.rmed.2008.10.004 
12. Cote C, Celli B. Pulmonary rehabilitation and the BODE index in COPD. Eur Respir J. 2005;26:630-636. doi:10.1183/09031936.05. 00045505

13. Ong K-C, Earnest A, Lu S-J. A multidimensional grading system (BODE index) as predictor of hospitalization for COPD. Chest. 2005;128:3810-3816. doi:10.1378/chest.128.6.3810

14. Disease GIfCOL. Global strategy for diagnosis, management and prevention of COPD (2015 update). 2015.

15. Vogelmeier CF, Criner GJ, Martinez FJ, et al. Global strategy for the diagnosis, management, and prevention of chronic obstructive lung disease 2017 report. GOLD executive summary. Am J Respir Crit Care Med. 2017;195:557-582. doi:10.1164/rccm.201701-0218PP

16. Barnes P, Celli B. Systemic manifestations and comorbidities of COPD. Eur Respir J. 2009;33:1165-1185. doi:10.1183/09031936.00 128008

17. Frei A, Muggensturm P, Putcha N, et al. Five comorbidities reflected the health status in patients with chronic obstructive pulmonary disease: the newly developed COMCOLD index. J Clin Epidemiol. 2014;67:904-911. doi:10.1016/j.jclinepi.2014.03.005

18. Sullivan SD, Ramsey SD, Lee TA. The economic burden of COPD. Chest. 2000;117:5S-9S. doi:10.1378/chest.117.2_suppl.5S

19. Dalal AA, Shah M, D’Souza AO, Rane PJ. Costs of COPD exacerbations in the emergency department and inpatient setting. Respir Med. 2011;105:454-460. doi:10.1016/j.rmed.2010.09.003

20. Cavaillès A, Brinchault-Rabin G, Dixmier A, et al. Comorbidities of COPD. Eur Respir Rev. 2013;22:454-475. doi:10.1183/09059180. 00008612
21. Celli B, MacNee W, Agusti A. ATS/ERS TASK FORCE Standards for the diagnosis and treatment of patients with COPD: a summary of the ATS/ERS position paper. Eur Respir J. 2004;23:932-946. doi:10. 1183/09031936.04.00014304

22. Johansson G, Mushnikov V, Bäckström T, et al. Exacerbations and healthcare resource utilization among COPD patients in a Swedish registry-based nation-wide study. BMC Pulm Med. 2018;18:17. doi:10.1186/s12890-018-0573-0

23. Pauwels RA, Buist AS, Calverley PM, Jenkins CR, Hurd SS, Medicine CC. Global strategy for the diagnosis, management, and prevention of chronic obstructive pulmonary disease: NHLBI/WHO Global Initiative for Chronic Obstructive Lung Disease (GOLD) Workshop summary. Am J Respir Crit Care Med. 2001;163:12561276. doi:10.1164/ajrccm.163.5.2101039

24. García-Polo C, Alcázar-Navarrete B, Ruiz-Iturriaga LA, et al. Factors associated with high healthcare resource utilisation among COPD patients. Respir Med. 2012;106:1734-1742. doi:10.1016/j.rmed.2012. 09.009

25. Dal Negro R, Celli B. Patient Related Outcomes-BODE (PROBODE): a composite index incorporating health utilization resources predicts mortality and economic cost of COPD in real life. Respir Med. 2017;131:175-178. doi:10.1016/j.rmed.2017.08.019

26. Bu X, Yang T, Thompson M, Hutchinson AF, Irving L. Changes in the BODE index, exacerbation duration and hospitalisation in a cohort of COPD patients. Singapore Med J. 2011;52:894-900.

\section{Publish your work in this journal}

The International Journal of COPD is an international, peer-reviewed journal of therapeutics and pharmacology focusing on concise rapid reporting of clinical studies and reviews in COPD. Special focus is given to the pathophysiological processes underlying the disease, intervention programs, patient focused education, and self managemen protocols. This journal is indexed on PubMed Central, MedLine and CAS. The manuscript management system is completely online and includes a very quick and fair peer-review system, which is all easy to use. Visit http://www.dovepress.com/testimonials.php to read real quotes from published authors. 